\title{
Critical notice: Darwinian reductionism
}

\author{
Marcel Weber
}

Received: 17 July 2007/ Accepted: 1 August 2007/Published online: 25 August 2007

(C) Springer Science+Business Media B.V. 2007

\begin{abstract}
This notice provides a critical discussion of some of the issues from Alex Rosenberg's Darwinian Reductionism, in particular proper functions and the relationship of proximate and ultimate biology, developmental programs and genocentrism, biological laws, the principle of natural selection as a fundamental law, genetic determinism, and the definition of "reductionism."
\end{abstract}

Keywords Reductionism - Proper functions - Ultimate vs. proximate biology · Biological laws · Developmental programs · Genocentrism · Principle of natural selection · Genetic determinism

\section{Introduction}

Reductionism has not had a good press in recent years, at least in philosophical journals. Meanwhile, biologists have been getting on with the task of explaining more and more biological phenomena in molecular terms, especially development. In the history of philosophy, antireductionism about biology is certainly not lacking in precedent; Aristotle and Kant are just two among many great philosophers who have defended anti-reductionistic views about the science of life. Therefore, from a historical perspective, the spectacular successes of molecular biology must stand as a major challenge to large and important parts of the philosophical tradition. In his ambitious new book, Alex Rosenberg sets out to prove that the philosophy of biology has been caught up in an "untenable dualism" and suggests a way out of it.

Alex Rosenberg, Darwinian Reductionism. Or, How to Stop Worrying and Love Molecular Biology. Chicago: The University of Chicago Press. 2006. Cloth \$40.00spec ISBN: 978-0-226-72729-5 (ISBN-10: 0-226-72729-7)

M. Weber $(\bowtie)$

Science Studies Program and Philosophy Department, University of Basel, Missionsstrasse 21, Basel 4003, Switzerland

e-mail: marcel.weber@unibas.ch 
I shall first present a brief synopsis of the book, before I single out some specific issues for critical discussion.

Darwinian Reductionism begins with a chapter titled "What Was Reductionism?" It summarizes some of the debates that have taken place since the heyday of logical empiricism and places them in the context of molecular biology. Fortunately, Rosenberg centers his discussion around molecular developmental biology rather than that old saw known as the reduction of classical genetics to molecular biology. There is, however, an excellent discussion of Kitcher's well-known argument that classical genetics provided full explanations of phenomena such as independent assortment (Kitcher 1984)—explanations that not only cited no molecular details, but would lose if they did. Rosenberg dismisses this argument as "explanatory Protagoreanism": Just because this kind of explanation appeals to our understanding does not mean that there is no deeper, microreductive explanation. The critique is rich and sophisticated and involves considerations on the pragmatics of explanation. Rosenberg also argues in Chapter 1 that the whole reductionism debate needs to be reconfigured. In logical empiricism, reduction meant the derivation of laws of some special science from more fundamental laws. This question is irrelevant, Rosenberg claims, because there are almost no laws in biology (see below for why it's only "almost" no laws). Instead, he argues, reductionists and antireductionists alike must come to terms with the fact that

biology is unavoidably terrestrial [...]. Thus, the debate between reductionists and antireductionists will have to be one about the explanation of particular historical facts, some obtaining for longer than others, but all of them ultimately the contingent results of general laws of natural selection operating on boundary conditions (4041).

With the exception of natural selection, Rosenberg claims, there are no general theories in biology, so the old notion of theory reduction is irrelevant.

The book continues, in Chapters 2 and 3, with an in-depth account of recent molecular developmental biology. After denying that pre-molecular developmental biology discovered more than a couple of "dormitive virtues" (explanatorily empty dispositions such as "organizers"), Rosenberg turns to the great late-20th century findings on development in the early Drosophila embryo. He describes these findings in terms of "reverse-engineering a piece of hardware to extract the software that it implements" (61). The discussion centers on theoretical accounts of regulatory gene networks that construe the control of the developmental process in terms of Boolean switching rules. This is not only analogous to a machine program, it is one, according to Rosenberg. The genes literally program development. At the same time, Rosenberg thinks that all talk of genetic information is metaphorical. Nonetheless, by claiming that there are literally genetic programs, he commits himself to the view that genes play some rather privileged role in development, a claim that many authors reject (e.g., Griffiths and Gray 1994; Sterelny and Griffiths 1999; Robert 2004).

In the next three chapters, 4-6, Rosenberg argues that there is only one true law in biology. Rosenberg calls it the "principle of natural selection (PNS)" and gives it different formulations, for example (160):

$(x)(y)(E)$ [If $x$ and $y$ are competing organisms in generation $n$, and $x$ is fitter than $y$ in $E$, then probably (there is some generation $n^{\prime}$, at which $x$ has more descendants than y)] 
There are alternative formulations, and Rosenberg is aware that this may not be the most general way of stating the principle. ${ }^{1}$ Rosenberg takes this to be an empirical law (in contrast to Sober 1993, who thinks that it's a priori) and he understands fitness in terms of a probabilistic propensity.

Now for what is probably Rosenberg's boldest claim in this book: He argues that the PNS is a physical law, or perhaps a chemical law (or both). Rosenberg needs this claim in order to make "natural selection safe for reductionism". I shall critically discuss this claim below.

The final two chapters (7-8) attempt to extend the reductionism defended to explanations of human behavior, in particular social cooperation. Here, Rosenberg's goal is to show that, far from supporting genetic determinism, reductionism and the associated research strategies of molecular biology have refuted genetic determinism. I shall provide a critical assessment of this claim as well.

As should be clear from this synopsis, this text has very high ambitions. I shall now begin to examine to what extent it can deliver on them.

\section{Proper functions and the relationship of ultimate and proximate biology}

Rosenberg firmly adheres to the view that "nothing in biology makes sense except in the light of evolution." Evolutionary biologists such as Mayr or Dobzhansky, who have defended this view, based their arguments on the assumption that a full understanding of organisms requires the identification of the ultimate causes of their characteristic properties. To use Mayr's favorite example, even if we fully understand the physiological mechanisms that induce migratory birds to flock together and embark on a long journey toward a warmer climate zone (i.e., the proximate cause), a full understanding of this behavior requires an account of what it was selected for in the birds' evolutionary past (i.e., the ultimate cause). On this received view, proximate and ultimate explanations are complementary and conceptually independent. This conceptual independence allows for the possibility of endorsing both reductionism about proximate biology and antireductionism about evolutionary biology. ${ }^{2}$

However, according to Rosenberg, ultimate and proximate biology are not conceptually independent. How could this be? Why can not biologists pinpoint an organism's molecular, physiological, developmental etc. mechanisms independently of its evolutionary history? For Rosenberg, this has to do with the way in which biologists pick the explananda, in other words, that which they want to explain by discovering the underlying mechanisms.

\footnotetext{
${ }^{1}$ It is certainly not, because as stated it is only applicable to populations with discrete generations. Evolutionary theorists use different fitness measures for populations with discrete generations and for agestructured populations with overlapping generations. In Weber (1998, Chapter 6), I argue that if there is a general principle of natural selection, then it is highly abstract and needs to be instantiated by specific models. On this view, the theory of natural selection is a family of models ("semantic view" of theories) and its content is not appropriately expressed by a universally quantified claim. Universally quantified claims only come in when it comes to stating classes of natural systems to which the models apply. The general theory is merely some sort of a guideline for building specific models. But I cannot prove that there is no universal formulation of the principle of natural selection in the manner in which Rosenberg envisions it.

2 The latter kind of antireductionism is usually justified on the grounds of the multiple realizability of fitness, see Sober (1984).
} 
Let us say, for example, that biologists want to understand how chick embryos form wings. "Wing" is a functional concept. In other words, the classification of some structure as a wing, including its exact delimitation from neighboring structures, involves an appeal to function (flight in this case). Rosenberg argues that the salient concept of function here must be that of proper function, that is, function as selected effect. A wing is a structure that was selected because it confers the ability to fly. It is a functional type, and "function" means proper function according to Rosenberg. The realizers of this functional type are heterogeneous because different structures with different evolutionary origins can confer the ability to fly. This is why there are also no natural kinds (essences) in the traditional sense in biology, Rosenberg argues. The upshot is that the way an organism is divided into parts crucially depends on the theory of natural selection. Since proximate biology takes its requests for explanation from such divisions ("what mechanisms control the development of the chick wing?"), it is conceptually dependent on evolutionary biology.

This position with respect to functions and proximate biology seems to put Rosenberg in the difficult position that, in order to maintain his reductionism, he must show either that the theory of natural selection is reducible to more fundamental theories or that it is itself a fundamental theory. He will end up choosing the second path. This will be discussed later; right now, there are some critical issues to be raised concerning Rosenberg's strong reliance on natural selection in the individuation of organic parts and the selection of molecular biology's explananda.

First of all, there will be difficulties in accounting for an important case: genes. If all biological kinds are defined via selected effects, Rosenberg must be careful not to make maladaptive or selectively neutral genes a conceptual impossibility. Perhaps, he could save his claim by arguing that genes form a kind not by virtue of all its members being beneficiaries of natural selection, but by virtue of the fact that what genes generally do (i.e., determine the linear sequence of proteins and RNA) being the reason why evolution has produced genes as a general kind. In other words, the coding property must have been directly selected for at some stage in evolution, which is a very strong empirical assumption.

This points to a second difficulty. Rosenberg's account of biological kinds requires biologists to know what various parts of an organism were selected for. In other words, it requires from them that, before they even understand the mechanisms that perform various tasks in the machinery of life, they must know what this task was selected for. This is tantamount to assuming a "God's eye view" of the evolutionary past of all organisms. ${ }^{3}$ However, in the laboratory, it seems, biologists rarely make assumptions about evolution. Surely, geneticists were classifying genes before they had any idea what they (as a general kind) had been selected for. Furthermore, there are clearly alternatives to how functional kinds are individuated in biology, for example, via hierarchies of contributing mechanisms (Craver 2001; Weber 2005b, 35-40) or via homology (Griffiths 2006).

Thirdly, Rosenberg's analysis also forgoes the possibility of giving non-adaptationist accounts of the evolution of form, for example, explanations that put developmental constraints and evolutionarily entrenched structures rather than adaptation by natural selection into the foreground (see Amundson 2005). Clearly, Rosenberg's book does have "adaptationism" written all over it. Whether or not some form of adaptationism or another is true is an empirical question; but it does not seem wise to exclude the alternatives on conceptual grounds.

\footnotetext{
${ }^{3}$ I owe this formulation to Daniel Sirtes.
} 


\section{Developmental programs and genocentrism}

According to popular accounts, DNA plays a special role in development because it carries "genetic information." This idea has been the target of much recent criticism (e.g., Sarkar 1996). If understood in a purely statistical sense, many biological structures could be said to carry information. If understood in a semantic or intentional sense, it commits us to the controversial idea that DNA has original intentionality, i.e., that it represents the phenotype (Sterelny and Griffiths 1999). Rosenberg wants to have none of this. He shows that DNA cannot be the bearer of intentional content because it is not sufficiently sensitive to substitutions of descriptions of its content. In other words, DNA does not show the phenomenon of referential opacity that many philosophers of mind see as the hallmark of intentionality. This is the best critique of the view that DNA bears intentional content that I have seen yet.

So Rosenberg rejects the attempt to base genocentrism on the idea that DNA bears information in the semantic sense. Instead, he argues that genocentrism is true because DNA, while not literally bearing informational states, nonetheless may be viewed as a program. He means this in the same sense as in "computer program". This is not in conflict with his views about intentionality and information, as computer programs have no original intentionality, i.e., intentionality that does not require an intentional agent who interprets the states in question.

Much of Rosenberg's case that there is literally a program that controls development in the Drosophila embryo is based on the work of Bodnar (1997). What Bodnar did is to integrate experimental findings on interactions between developmental genes (for instance, genes described by Christiane Nüsslein-Volhart and other experimentalists) into a computational model that can simulate the developmental process on a personal computer. In doing this, Bodnar drew on methods from chemistry and engineering. It turns out that most of the genes that control development can be represented as Boolean switches. That is, the switches are "on" or "off" depending on whether the concentrations of certain transcription factors are above or below a certain threshold. Bodnar then generalized a series of switching rules that generate the gene activation patterns actually observed in living embryos. He was even able to predict some interactions on the basis of engineering considerations.

There is certainly no denying that this theoretical and in silico work is impressive and an important addition to the experimentalists' work of painstakingly identifying genes and proteins that are involved in morphogenesis. However, what this does not prove is that a living Drosophila embryo literally executes a program, as Rosenberg thinks. It merely shows that parts of the genetic mechanisms of embryonic development can be modeled as if they were logical switches in a computer program. What's going on in the embryo is also describable in causal-mechanistic terms (Weber 2005b, Chapter 8). ${ }^{4}$ Causes, and perhaps mechanisms, belong to the ontological furniture of the world. Programs and calculations do not. Being a program is a relation between parts of a machine and the designer or user of that machine. The program is that part of the machine that its designer included in order to be able to control the machine's operations. Similarly, a computation is a process the input and output states of which are interpreted by an intentional user.

So, "program", "computation", and also "calculation" are intentionally laden notions (Weber 2005a). Since Rosenberg believes that there is no original intentionality in the

\footnotetext{
${ }^{4}$ Experimental biologists tend to think in causal-mechanical rather than in computational terms. Note that Rosenberg's main witness, J.W. Bodnar, is a computer scientist, not an experimental biologist.
} 
molecular processes that make up life, he is not entitled to apply these terms literally to a Drosophila embryo. While he might be too reductionistic when it comes to natural selection, Rosenberg is not reductionistic enough about developmental biology. His construal of Drosophila development looks more like machine functionalism in spirit, which is supposed to be a species of non-reductive physicalism.

Of course, these difficulties also put Rosenberg's genocentrism in limbo. While it is possible that there are deeper ontological (e.g., causal) reasons why development can be so successfully modeled in computational terms, he would need to spell out these reasons. For a reductionist, unless she holds the implausible view that genes carry original intentionality (see above), genocentrism can only be justified on strictly causal grounds (Weber 2005b, Chapter 8). Whether or not this is possible is beyond the scope of this essay review.

\section{Biological laws}

Rosenberg's argument against biological laws is not so much based on evolutionary contingency (as in Beatty 1995), but on the idea that there is nothing that could stop natural selection from producing highly heterogeneous kinds and that, therefore, it does. Natural selection is insensitive to how biological functions are implemented. For example, wings are solutions to design problems involving flight, but wings come in all kinds of different designs. Similarly, an enzymatic activity can be exerted by different proteins (or RNAs) and may involve chemically different catalytic mechanisms. On Rosenberg's account, this is why biological kinds are always heterogeneous. He seems to think that this already implies the non-existence of biological laws (except the law of natural selection, see Sect. "Natural selection as a fundamental law").

It seems to me that this conclusion is based on a particular understanding of laws, namely one that requires them to be generalizations that range across different species (or other taxonomic kinds). However, as Waters (1998) has convincingly argued, taxonomic kinds are not the appropriate domain of quantification for causal generalizations in biology. There may be relevant causally homogeneous kinds that are independent of the distribution of characters in the phylogenetic tree. We may extend this argument and say that there may be causally homogeneous biological kinds (which need not be structurally homogeneous) that are independent of selected effect-functions. Of course, Rosenberg will not recognize them, because he thinks that all biological kinds are constituted by selected effect-functions. As is well known, one philosopher's modus ponens is another philosopher's modus tollens. Some will conclude that if Rosenberg's theory of biological kinds doesn't allow for laws (or causal generalizations, which I take to be the same), so much the worse for Rosenberg's theory of biological kinds.

According to Hitchcock and Woodward (2003), there are causal generalizations that are distinguished not by supporting counterfactual claims about a heterogeneous array of objects, but about a variety of different conditions for a single system (or type of system). Imagine that there was only one species of animal where Hodgkin-Huxley action potentials occurred. Assume further that the behavior of the neural membranes of this animal were governed by certain causal regularities, namely those stated in the famous HodgkinHuxley equations. This would make these action potentials (or the mechanisms that produce them) a causally homogeneous kind. The laws in questions are derived laws that follow from fundamental physical laws (Coulomb's law, Second Law of Thermodynamics) in conjunction with certain boundary conditions (Weber 2005b, Chapter 2). Since these 
boundary conditions describe a type of biological system, we may speak of a biological law.

Now, I do not see why the nomological status of this generalization should be affected by the fact that Hodgkin-Huxley action potentials actually occur in a broad variety of different species, and are realized by structurally heterogeneous classes of ion channelsjust so long as they form a causally homogeneous class (which they do). Furthermore, these generalizations would be laws even if the ion channels involved were selected for different reasons in different species. They probably were not, but that is not my point. The point is that there are causal generalizations about biological kinds that are not taxonomic kinds nor selected effect-kinds. By allowing only generalizations that range over functional kinds, where functions are only of the etiological sort, Rosenberg impoverishes biology's explanatory repertoire.

\section{Natural selection as a fundamental law}

Because he assumes an all-pervasive role for natural selection in the constitution of biological kinds, Rosenberg needs to "make natural selection safe for reductionists." This could be done either by showing that the PNS reduces to fundamental laws, or that it is itself a fundamental law. Rosenberg chooses the second path. He construes the PNS (in some formulation, not necessarily his own) as a non-derived physical law. Thus, it is not a physical law like the Second Law of thermodynamics, which he considers derived. In other words, it is not a physical law that results once some other physical laws and some initial and boundary conditions are fixed. It is indeed a fundamental law of nature.

This claim may strike many as rather eccentric. Yet it must be admitted that this view opens a way out from what many authors perceive as an impasse: On the one hand, most philosophers accept that the physical facts fix all the biological facts, and that the physical domain is causally closed. Thus, the "ideal explanatory text" must always be a physical text, and no part of that text (e.g., one that is produced by selectively attending to some features of the full text) can provide a better explanation for a phenomenon than the full text. On the other hand, there appears to be at least one biological theory that is not reducible to physical theories and that seems to explain some facts better then any physical theory: the theory of natural selection. This seems to be in conflict with physicalism. How can this be? This Gordian knot can be cut with one stroke by simply declaring natural selection a non-derived physical law, thus removing the need of reducing it to anything more fundamental.

Obviously, this move creates the need of explaining why the PNS does not feature in physics textbooks, or why physicists have never felt the need of introducing it. In order to do so, Rosenberg offers quite an ingenuous story about self-replicating molecules, i.e., molecules that can catalyze reactions that result in the synthesis of more tokens of their type. He imagines scenarios where a number of such molecules compete for building blocks under changing environmental conditions. In order to increase in number, such molecules must strike some good balance between stability and self-replication (a molecule that is more stable than its competitors has a tendency to make it on the long term, unless the less stable competitors produce copies at a faster rate). This is beginning to sound like biological fitness. Add to this the possibility of a heterogeneous environment where some stability/replication regime might win out on the long run, and you basically have natural selection. So natural selection explains some physical facts without being derivable from more fundamental laws. 
The reason why we do not find natural selection in physical chemistry textbooks, according to Rosenberg, is basically scale in time and space. Physicists and chemists do not normally study the distribution of molecules over longer time scales, or in different locations and heterogeneous environments. So natural selection as a physical law must be added to the others as soon as this kind of question is being asked.

Rosenberg then argues that such a PNS for molecules is not derivable from physical-chemical laws because there is always an infinity of different combinations of replicability and stability as well as chemical environments that might push a certain type of molecules to victory on the long run. The laws of physics need specific initial and boundary conditions to explain the distribution of the molecules at some later time. The PNS can do the same for infinitely many different initial conditions.

This argument, ingenious as it is, raises the question if Rosenberg is not inadvertently inviting bedfellows he does not want. To me, this sounds a lot like what some antireductionists say about principles such as natural selection: they allow us to see the unity in physically extremely heterogeneous kinds of processes and are therefore autonomous, irreducible principles of some higher level (Sober 1984). Rosenberg tries to avoid this by saying that natural selection is not a principle at some higher level; it is one that operates at all levels. On the other hand, I do not think antireductionists would deny that natural selection can also act at the level of molecules. The difference between Rosenberg and these antireductionists is subtle: For Rosenberg, only the principle for molecules is nonderived; principles of natural selection for higher levels are in principle derivable from principles that operate at a lower level.

\section{Genetic determinism}

While lay persons may be inclined to see genetic determinism as a species of reductionism, Rosenberg wants to show that the relentless pursuit of reductionistic research strategies in recent biology has actually refuted genetic determinism.

The two main goals of the final two chapters are as follows: First, Rosenberg wants to show that a combination of evolutionary theory with genomics has established that human cooperation is not an adaptation that maximized inclusive fitness (a fitness measure that takes the contribution of relatives to the propagation of gene tokens into account). It is rather a fortuitous result of the exercise of other behavioral dispositions. Rosenberg contends that "[t]here is little reason to suppose that any human activity as complex as social cooperation could have an interesting genetic basis" (208). One reason that he cites in favor of this claim is that there appear to be no relevant behavioral traits that are controlled by single genetic loci like, for instance, the ability to roll the tongue in humans. Second, Rosenberg points out that our ancestors have ceased to live in groups where there could be selection for kin altruism long ago.

Both of these arguments strike me as non-sequiturs. Traits can be genetic without there being a single locus that controls them. Further, some trait can have a genetic basis even if natural selection has eliminated all variation in this trait. So even if there is currently no proof of genetic variation in human traits related to social behavior, this does not imply that there has not been selection for such genes in the past. As for the other argument, kin altruism is not the only possible cause of social cooperation. There could have been other evolutionary forces at work, for instance, frequency-dependent selection (which is the subject of evolutionary game theory) or group selection. Rosenberg seems to think that cooperation in humans must have arisen by cultural evolution. If his arguments were 
sound, there would only be a handful of species that could engage in social behavior (namely, those that are capable of cultural transmission of traits). But there are clearly many more species that have evolved cooperative behavior.

Overall, Rosenberg's picture of the evolution of human cooperation does not seem very plausible to me. What should these other behavioral dispositions that he alludes to have evolved for? And what about those human capacities that subserve social cooperation, such as language, emotions, the theory of mind, and perhaps also self-consciousness? What should these be if not adaptations for cooperation in a species that already engaged in complex social behaviors?

A more convincing case is Rosenberg's discussion of phenylalanine ketonuria (PKU), which is generally known as an inborn error of metabolism. Here, molecular studies appear to have revealed that PKU is not a single condition. Instead, it seems to be a whole complex of diseases, some of which can occur even in the absence of defective alleles of the corresponding gene. Obviously, only molecular research had any chance of unveiling this complexity. Whatever one means precisely by "genetic determinism" - and it has, I think, always been a rather vague notion-only reductionistic research strategies can pinpoint the multiplicity of causes that may be responsible for a given medical condition.

\section{What is (Darwinian) reductionism?}

Rosenberg spends much time explaining what reductionism was, but not that much in saying what it is. Here is his in nuce account (4):

Reductionism is the thesis that biological theories and the explanations that employ them do need to be grounded in molecular biology and ultimately physical science, for it is only by doing so that they can be improved, corrected, strengthened, made more accurate and more adequate, and completed.

Clearly, this is a controversial claim, as many philosophers have tried to defend some sort of autonomy, for example, for evolutionary theory and its population-level explanations. It is also clear that Rosenberg wants to flatly deny this autonomy. However, one would wish to know more about how this "grounding" of all biology in molecular science and physical theory is supposed to work. On the one hand, Rosenberg seems to reject derivational models of reduction. On the other hand, when discussing the PNS, he often appeals to "in principle derivability." For example (193):

[A]t each level of the organization of matter, there turns out to be a PNS (M.W.) and each one should be in principle derivable from the PNS for the immediately lower level or some other lower level(s), all the way back down to the PNS for molecules.

So are we back to derivational reduction after all?

Taking stock, this book is more than a novel defense of reductionism. As a positive contribution to the classical reductionism debate, it suffers a bit from the lack of an explicit account of reduction. Nonetheless, it contains penetrating critiques of some standard antireductionist arguments that antireductionists will need to deal with. But what probably distinguishes this book most is that it contains a general philosophical theory of the whole of biological science the likes of which I have hardly seen before, at least in terms of breadth, boldness, and coherence. Not many will agree with its content, not even fellow reductionists. Many philosophers of science will question the very possibility of such a general theory and confront Rosenberg's account with one version of scientific pluralism or 
another. But in the interest of a philosophical pluralism, it is important that not everyone give up the search for a general theory of scientific knowledge. For all those who care about the philosophy of biology - no matter whether they are reductionists, pluralists, or anything in between - the new Rosenberg is an absolute "must read."

\section{References}

Amundson R (2005) The changing role of the embryo in evolutionary biology: roots of Evo-Devo. Cambridge University Press, Cambridge

Beatty J (1995) The evolutionary contingency thesis. In: Wolters G, Lennox JG, McLaughlin P (eds) Concepts, theories, and rationality in the biological sciences. University of Pittsburgh Press, Pittsburgh, pp 45-81

Bodnar JW (1997) Programming the Drosophila embryo. J Theor Biol 188:391-445

Craver CF (2001) Role functions, mechanisms, and hierarchy. Philos Sci 68:53-74

Griffiths PE (2006) Function, homology and character individuation. Philos Sci 73:1-25

Griffiths PE, Gray RD (1994) Developmental systems and evolutionary explanation. J Philos 91:277-304

Hitchcock C, Woodward J (2003) Explanatory generalizations, part II: plumbing explanatory depth. Nous 37:181-199

Kitcher P (1984) 1953 and all that. A tale of two sciences. Philos Rev 93:335-373

Robert JS (2004) Embyrology, epigenesis, and evolution: taking development seriously. Cambridge University Press, Cambridge

Sarkar S (1996) Biological information: a sceptical look at some central dogmas of molecular biology. In: Sarkar S (ed) The philosophy and history of molecular biology: new perspectives. Kluwer, Dordrecht, pp 187-231

Sober E (1984) The nature of selection. Evolutionary theory in philosophical focus. MIT Press, Cambridge

Sober E (1993) Philosophy of biology. Oxford University Press, Oxford

Sterelny K, Griffiths PE (1999) Sex and death: an introduction to philosophy of biology. The University of Chicago Press, Chicago

Waters CK (1998) Causal regularities in the biological world of contingent distributions. Biol Philos 13:536

Weber M (1998) Die Architektur der Synthese. Entstehung und Philosophie der modernen Evolutionstheorie. Walter de Gruyter, Berlin

Weber M (2005a) Genes, causation and intentionality. Hist Philos Life Sci 27:399-411

Weber M (2005b) Philosophy of experimental biology. Cambridge University Press, Cambridge 